University of Nebraska - Lincoln

DigitalCommons@University of Nebraska - Lincoln

$9-1-2005$

\title{
Simultaneous elastic and electromechanical imaging by scanning probe microscopy: Theory and applications to ferroelectric and biological materials
}

J. Shin

University of Tennessee

B. J. Rodriguez

North Carolina State University, Raleigh, brian.rodriguez@ucd.ie

Arthur P. Baddorf

Oak Ridge National Laboratory, baddorfap@ornl.gov

T. Thundat

Oak Ridge National Laboratory, Oak Ridge, Tennessee

E. Karapetian

Suffolk University, Boston, Massachusetts

See next page for additional authors

Follow this and additional works at: https://digitalcommons.unl.edu/physicsgruverman

Part of the Physics Commons

Shin, J.; Rodriguez, B. J.; Baddorf, Arthur P.; Thundat, T.; Karapetian, E.; Kachanov, M.; Gruverman, Alexei; and Kalinin, Sergei V., "Simultaneous elastic and electromechanical imaging by scanning probe microscopy: Theory and applications to ferroelectric and biological materials" (2005). Alexei Gruverman Publications. 37.

https://digitalcommons.unl.edu/physicsgruverman/37

This Article is brought to you for free and open access by the Research Papers in Physics and Astronomy at DigitalCommons@University of Nebraska - Lincoln. It has been accepted for inclusion in Alexei Gruverman Publications by an authorized administrator of DigitalCommons@University of Nebraska - Lincoln. 


\section{Authors}

J. Shin, B. J. Rodriguez, Arthur P. Baddorf, T. Thundat, E. Karapetian, M. Kachanov, Alexei Gruverman, and Sergei V. Kalinin 


\title{
Simultaneous elastic and electromechanical imaging by scanning probe microscopy: Theory and applications to ferroelectric and biological materials
}

\author{
J. Shin \\ Condensed Matter Sciences Division, Oak Ridge National Laboratory, Oak Ridge, Tennessee 37831 \\ and Department of Physics and Astronomy, The University of Tennessee, Knoxville, Tennessee 37996 \\ B. J. Rodriguez \\ Department of Physics, North Carolina State University, Raleigh, North Carolina 27695 \\ A. P. Baddorf \\ Condensed Matter Sciences Division, Oak Ridge National Laboratory, Oak Ridge, Tennessee 37831 \\ T. Thundat \\ Department of Physics and Astronomy, The University of Tennessee, Knoxville, Tennessee 37996 \\ and Life Sciences Division, Oak Ridge National Laboratory, Oak Ridge, Tennessee 37831 \\ E. Karapetian \\ Department of Mathematics and Computer Science, Suffolk University, Boston, Massachusetts 02114 \\ M. Kachanov \\ Department of Mechanical Engineering, Tufts University, Medford, Massachusetts 02155
}

A. Gruverman

Department of Materials Science and Engineering, North Carolina State University, Raleigh, North Carolina 27695

S. V. Kalinin ${ }^{\text {a) }}$

Condensed Matter Sciences Division, Oak Ridge National Laboratory, Oak Ridge, Tennessee 37831

(Received 3 March 2005; accepted 15 August 2005; published 20 September 2005)

\begin{abstract}
An approach for combined imaging of elastic and electromechanical properties of materials, referred to as piezoacoustic scanning probe microscopy (PA-SPM), is presented. Applicability of this technique for elastic and electromechanical imaging with nanoscale resolution in such dissimilar materials as ferroelectrics and biological tissues is demonstrated. The PA-SPM signal formation is analyzed based on the theory of nanoelectromechanics of piezoelectric indentation and signal sensitivity to materials properties and imaging conditions. It is shown that simultaneous measurements of local indentation stiffness and indentation piezocoefficient provide the most complete description of the local electroelastic properties for transversally isotropic materials, thus making piezoacoustic SPM a comprehensive imaging and analysis tool. The contrast formation mechanism in the low frequency regime is described in terms of tip-surface contact mechanics. Signal generation volumes for electromechanical and elastic signals are determined and relative sensitivity of piezoresponse force microscopy (PFM) and atomic force acoustic microscopy (AFAM) for topographic cross-talk is established. (C) 2005 American Vacuum Society.
\end{abstract}

[DOI: $10.1116 / 1.2052714$ ]

Physical properties of functional materials such as ferroelectric ceramics and polymers, piezoelectric polymer composites and biological tissues are determined by complex electrical and electromechanical interactions on the length scales from hundreds of microns to few nanometers. While macroscopic properties are readily accessible by conventional indentation and piezoelectric characterization techniques, until recently mechanical and especially electromechanical behavior at the nanoscale was experimentally unattainable. Understanding the origins of electromechanical and mechanical functionality in complex materials and bio-

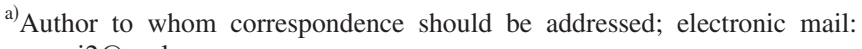
sergei2@ornl.gov logical systems requires the ability to probe elastic and electromechanical properties on the level of individual nanoscale structural elements. In the last decade, several SPM techniques including atomic force acoustic microscopy (AFAM), ${ }^{1}$ scanning local acceleration microscopy, ${ }^{2}$ force modulation microscopy, ${ }^{3}$ hybrid nanoindentation, ${ }^{4}$ and ultrasonic force microscopy (UFM), ${ }^{5}$ were developed to study the elastic properties of material on the nanoscale. Even conventional intermittent mode atomic force microscopy provides a wealth of information on the local mechanical properties in the phase image. ${ }^{6}$ In parallel, a number of SPM techniques, most notably piezoresponse force microscopy (PFM), ${ }^{7}$ were developed to address the local electromechanical properties and their applicability for biological systems was recently 
illustrated. ${ }^{8}$ The image formation mechanism and hence materials contrast in these SPMs is ultimately determined by complex contact mechanics of tip-surface interactions. To date, the tip-surface contact is typically modeled using a simple spherical or power law Hertzian model for isotropic materials along with modifications that include capillary and van der Waals force effects. However, this level of approximation is insufficient for understanding SPM data on piezoelectric materials that possess strong electromechanical coupling. The bias-dependent contact mechanics of the tipsurface junction and the signal generation volume and resolution of mechanical and electromechanical SPMs has until recently been unknown.

Recently, a quantitative theoretical description of nanoelectromechanics of tip-surface interaction for several tip geometries was developed. ${ }^{9,10}$ This description has shown that for transversally isotropic piezoelectric material (e.g., $c^{+}, c^{-}$ domains in tetragonal perovskites) characterized by 10 independent electroelastic constants, the tip-surface contact mechanics can be described by stiffness equations relating the indentation force, tip bias, indentation depth, and materials properties, providing an extension of Hertzian contact mechanics. For all simple tip geometries, materials properties are described by only three parameters, indentation elastic stiffness, $C_{1}^{*}$, indentation piezocoefficient, $C_{3}^{*}$, and indentation dielectric constant, $C_{4}^{*}$. AFAM and UFM response is determined by $C_{1}^{*}$, while PFM is sensitive to $C_{3}^{*} / C_{1}^{*} \cdot{ }^{10}$ All indentation stiffnesses are complex functions of electroelastic constants of material, $C_{i}^{*}=C_{i}^{*}\left(c_{i j}, e_{i j}, \varepsilon_{i j}\right)$, where $c_{i j}$ are elastic stiffnesses, $e_{i j}$ are piezoelectric constants, and $\varepsilon_{i j}$ are dielectric constants. Thus, the maximum information on electroelastic properties for a transversally isotropic material that can be obtained from a SPM experiment is given by these three quantities. Consequently, mapping of $C_{i}^{*}$ distributions provides a comprehensive image of surface electroelastic properties.

Both imaging and quantitative interpretation of SPM data on complex materials systems require development of SPM techniques simultaneously sensitive to elastic, electromechanical, and electrical properties. Recently, Rabe et al. ${ }^{11}$ has demonstrated an approach for sequential AFAM and PFM imaging using topographic markers. Here, we demonstrate simultaneous elastic and electromechanical SPM imaging, referred to as piezoacoustic SPM (PA-SPM), and illustrate its applicability to several materials systems. The SPM signal formation is analyzed based on the theory of nanoelectromechanics of piezoelectric indentation. Signal generation volumes and resolution for elastic and electromechanical property measurements are derived. Given that the dielectric properties of the tip-surface junction generally cannot be accessed due to the smallness of the corresponding capacitance, the PA-SPM approach provides the maximum possible information on local electroelastic properties for transversally isotropic material that can be achieved in SPM experiment.

PA-SPM is implemented on a commercial SPM system (Veeco MultiMode NS-IIIA) equipped with additional func-

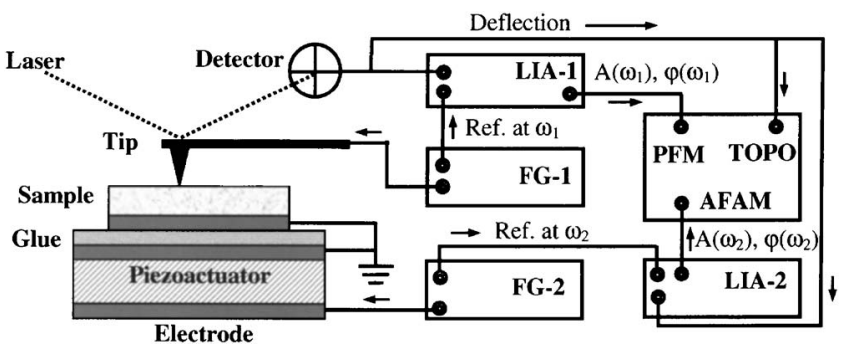

FIG. 1. Experimental set-up for piezoacoustic SPM. Function generator FG-1 biases the tip and bias-induced tip deflection measured by lock-in amplifier LIA-1 constitutes the PFM signal. Function generator FG-2 biases the piezoactuator and vibration induced tip deflection measured by lock-in amplifier LIA-2 constitutes the AFAM signal.

tion generators and lock-in amplifiers (DS 345 and SRS 830, Stanford Research Instruments, and Model 7280, Signal Recovery) as shown schematically in Fig. 1. For AFAM measurements, the samples were glued to a commercial lead zirconium titanate (PZT) oscillator. ${ }^{12}$ To minimize cross-talk between PFM and AFAM signals, the top electrode was always grounded and a modulation bias, $V_{2}$, at frequency $\omega_{2}$, was applied to the bottom electrode. Biasing the top electrode results in the potential drop between the tip and the surface, providing a strong piezoelectric contribution to acoustic signal. For PFM measurements, a custom-built sample holder was used to allow direct tip biasing and to avoid capacitive cross-talk in the SPM electronics. Alternatively, for ferroelectric thin film samples, a PFM modulation bias was applied to the sample bottom electrode, electrically disconnected from the top electrode of the actuator. The PFM modulation amplitude was $V_{1}$, at frequency $\omega_{1}$. Frequencies $\omega_{1}$ and $\omega_{2}$ are selected such that to avoid the overlap between higher and lower overtones. Typical values were $\omega_{1}$ $=99 \mathrm{kHz}$ and $\omega_{2}=70 \mathrm{kHz}$, though in some cases AFAM or PFM measurements were performed in the $1-2 \mathrm{MHz}$ range to utilize the dynamic stiffening effect. Measurements were performed using Pt and Au coated tips (NCSC-12 C, Micromasch, $l \approx 130 \mu \mathrm{m}$, resonant frequency $\sim 150 \mathrm{kHz}$, spring constant $k \sim 4.5 \mathrm{~N} / \mathrm{m}$ ), which were found to provide optimal performance in PFM measurements. To maximize AFAM contrast, measurements were performed with softer cantilevers (NCSC-36 C, Micromasch, $l \approx 130 \mu \mathrm{m}$, resonant frequency $\sim 70 \mathrm{kHz}$, spring constant $k \sim 0.5 \mathrm{~N} / \mathrm{m})$. Lock-in amplifiers were used to determine the magnitude and phase of the cantilever response at the frequencies of modulation signals. The output amplitudes, $A_{n}$, and phase shift, $\theta_{n}$, where $n=1,2$ corresponds electrical to and mechanical excitations, respectively, are recorded by the SPM system electronics. This experimental set-up also allows acquisition of additional data (e.g., mixed frequency signal between mechanical and electrical modulations or higher harmonics of tip response) providing information on non-linear electroelastic behavior in tip-surface junction. To determine the absolute magnitude of electromechanical response, the vertical photodiode sensitivity was estimated and the lock-in signal output was converted to the units of $\mathrm{pm} / \mathrm{V}$. Given that PFM signal is fairly insensitive to the details of tip-surface junction geom- 


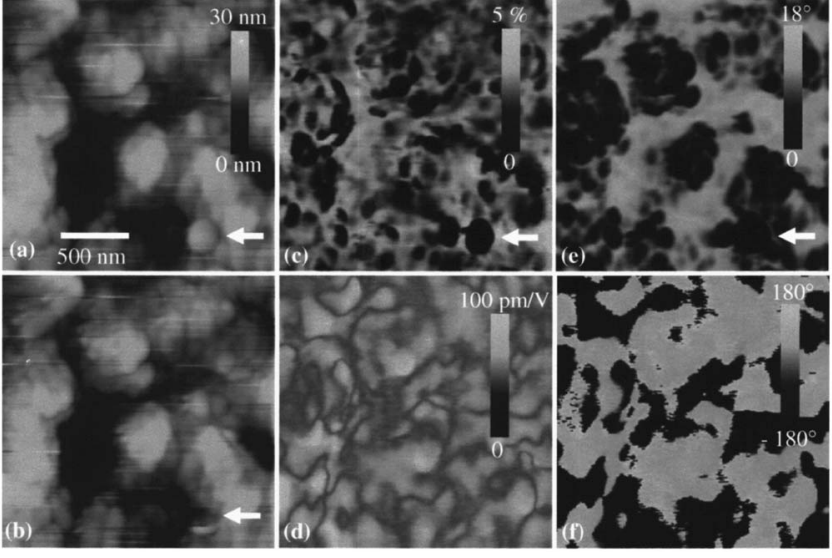

FIG. 2. Surface topography (a,b) AFAM amplitude (c), PFM amplitude (d), AFAM phase (e), and PFM phase (f) images on a PZT surface. Images are acquired consecutively; however, both modulations were present during the acquisition.

etry as discussed below, these numbers yields a reliable estimate of the electromechanical response of the surface. For AFAM, quantitative signal interpretation in terms of effective spring constant of tip-surface junction is extremely complex task that requires determination of several characteristic resonance frequencies at each image point. ${ }^{13}$ Furthermore, calculation of the effective Young's modulus of the material requires the knowledge of tip geometry, and also renders this technique sensitive to topographic cross-talk, as will be illustrated below. Therefore, we did not attempt to quantify AFAM data, limiting most cases to qualitative observations. More detailed frequency-detection AFAM studies on biomaterials will be reported elsewhere. ${ }^{14}$

To illustrate the validity of this approach, Fig. 2 illustrates the surface topography and PFM and AFAM images of a sol-gel PZT thin film. The low-frequency AFAM amplitude image [Fig. 2(c)] exhibits a number of dark spots indicative of a lower local Young's modulus of the surface, either due to the presence of local contaminates or due to a low-density grain structure. Note that some topographical features [arrow in Figs. 2(a) and 2(b)] change during consecutive scans, indicative of a contaminant particle. The average signal variation in the AFAM amplitude image is $\sim 1 \%$ with maximal signal deviation (at the dark spots) from an average of $\sim 4 \%$. Note that the AFAM phase image [Fig. 2(e)] also demonstrates spots at the same locations. There is a clear correlation between topographic, amplitude, and phase images, suggesting a significant cross-talk between the three. This can be expected, since the contact stiffness of the tip-surface junction that defines the AFAM contrast is strongly dependent both on the local curvature of the surface (which changes the effective contact radius) and the local slope (which changes the normal force component). Despite this, the images show clear differences in relative intensities, positions, and apparent resolution, of features indicative of complementary information. Shown in comparison are PFM amplitude [Fig. 2(d)] and phase [Fig. 2(f)] images of the same region. The signal strength is of the order of $\sim 50 \mathrm{pm} / \mathrm{V}$, as expected for

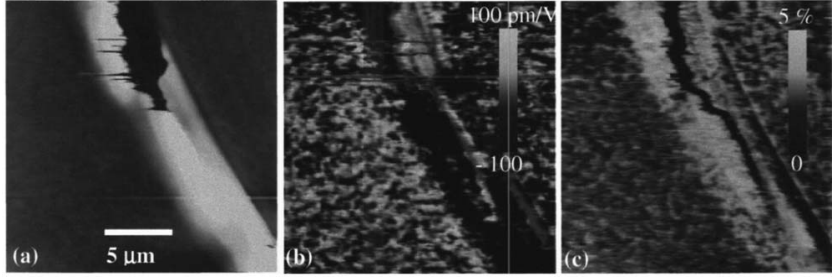

FIG. 3. Surface topography (a), PFM $x$-signal (b), and AFAM phase (c) images of the crack region on the PZT thin-film surface.

strongly piezoelectric material such as PZT. Contrast in the PFM amplitude indicates regions with different crystallographic orientation and/or surface composition. Amplitude minima at the domain walls are clearly observed. The PFM phase image shows $180^{\circ}$ phase changes at domain walls due to variation in polarization orientation. In this case, the PFM and AFAM images were acquired sequentially due to the limitations imposed by the number of available input channels on detection equipment. However, both modulations were active during image acquisition.

PFM and AFAM images of the crack regions in the PZT thin film are shown in Fig. 3. Here, the mixed PFM signal, $P R=A_{1} \sin \theta_{1}$, and the AFAM phase signal are acquired simultaneously. Note that the AFAM image is uniformly bright around the crack, implying a partial material debonding from the substrate. At the same time, PFM shows a distinct contrast in different parts of the crack region, suggesting that mechanical stress has resulted in partial polarization switching.

PA-SPM provides a unique opportunity in accessing biomechanical and bioelectromechanical properties of biological tissues. It is well known that biological materials are often elastically inhomogeneous and are comprised of regions with different elastic properties. Moreover, collagen, a major component of human and animal bone tissues, is strongly piezoelectric. ${ }^{15-18}$ A challenge in imaging of biological systems is that local elastic properties can potentially affect electromechanical response in SPM experiments. Moreover, lower indentation moduli as compared to inorganic ferroelectrics result in relatively larger electrostatic force contribution to the measured piezoresponse. Simultaneous imaging of both responses in PA-SPM addresses this problem. Illustrated in Figs. 4 and 5 are local topography, PFM and AFAM images of a polished human tooth surface at the dentine-enamel junction (Fig. 4) and in the pulp region (Fig. 5). The topographic image in Fig. 4(a) clearly illustrates the $\sim 300 \mathrm{~nm}$ grains forming a dense dentine layer. Corresponding AFAM images clearly show bright regions between the grains, either due to the organic layers between the hydroxyapatite grains or topographic cross-talk. Some of the grains exhibiting contrast variations between the grains can be seen due to a difference in mechanical properties of individual grains. The complementary PFM image clearly shows electromechanical activity in several regions. The characteristic response signal is of the order of $\sim 3-5 \mathrm{pm} / \mathrm{V}$, i.e., more than an order of magnitude smaller than for inorganic 


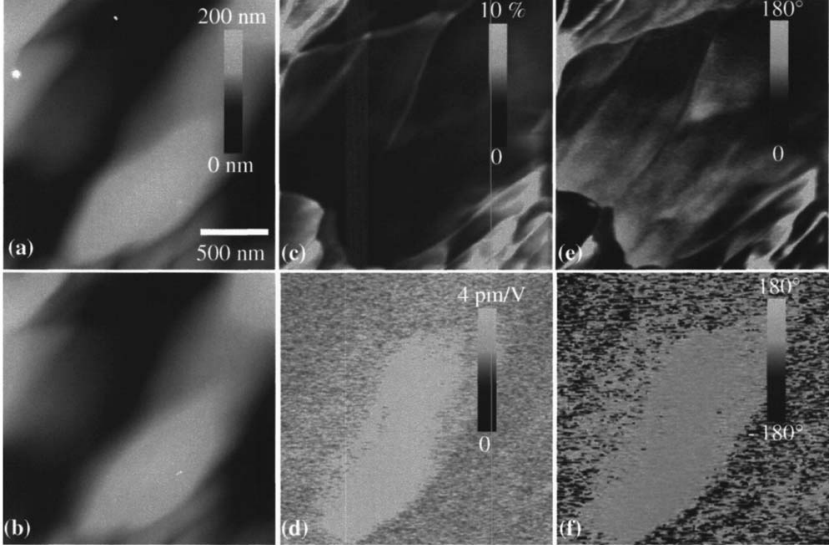

FIG. 4. Surface topography (a,b) AFAM amplitude (c), PFM amplitude (d), AFAM phase (e), and PFM phase (f) images on the dentine region of a human tooth

piezoelectric materials such as PZT. Surface topography and electromechanical properties of the pulp region are shown in Fig. 5. Here, the AFAM image shows completely different grain morphology, while there is no significant electromechanical activity in piezoresponse image.

As a second example, shown in Fig. 6 are surface topography and electromechanical response images of a crosssectioned antler. In this case, AFAM images (not shown) are relatively featureless and contain a number of features that can be attributed to topographic crosstalk. In comparison, large scale PFM images show that the surface exhibits nonzero electromechanical contrast. The contrast is virtually uniform along the surface, and thus can be attributed to either noise or small piezoelectric domains. Also note that significant PFM contrast variations can be observed only in the vicinity of the pore (canaliculi) on the surface with zero signal in the center and enhanced PFM amplitude at the circumference [Figs. 6(b) and 6(c)]. This observation can be rationalized as following. The large topographic features can be associated with enhanced PFM contrast, presumably due to the tip side touching the pore wall or orientation dependence

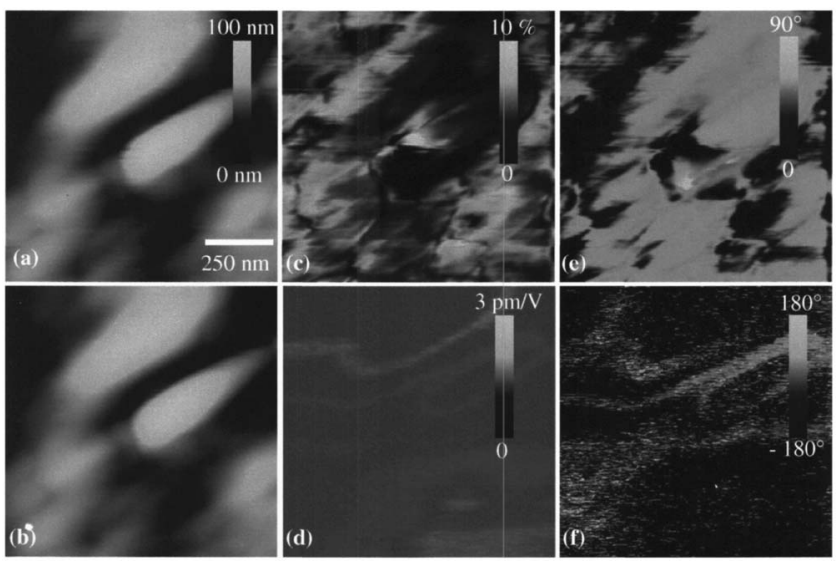

FIG. 5. Surface topography (a,b) AFAM amplitude (c), PFM amplitude (d), AFAM phase (e), and PFM phase (f) images on the pulp region of a human tooth.

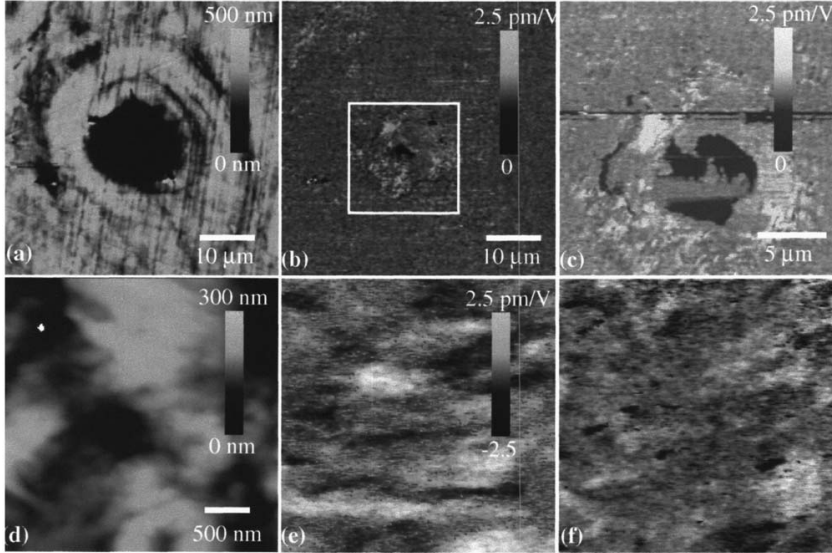

FIG. 6. Surface topography (a) and vertical PFM amplitude (b,c) images of cross-sectioned deer antler in the vicinity of the pore. (d) Surface topography and high resolution (e) vertical and (f) lateral PFM $x$-signal images of a deer antler.

of piezoresponse (shear piezoelectricity), while in the center the absence of tip apex-surface contact produces an imaging artifact. Note the similarity between Figs. 6(b) and 6(c) and previous studies on PFM in bones. ${ }^{8}$ The intrinsic PFM in antler can be visualized only in high resolution images as shown in Figs. 6(e) and 6(f), illustrating the presence of both vertical and lateral PFM signal, which are not correlated with surface topographic features, thus confirming its true electromechnical origin. Similarly to Fig. 4, the characteristic PFM response is $\sim 3 \mathrm{pm} / \mathrm{V}$, as can be expected for collagenous material and comparable to the macroscopic response. ${ }^{16}$

For both PZT and biological materials, the measured local PFM response cannot be compared directly to calculated value, since the crystallographic orientation (and materials properties for collagen) are not well known. However, it has been shown in Ref. 9 that the PFM signal is numerically close to the effective piezoelectric coefficient $d_{33}$ of the material. Experimentally measured values for PZT $(\sim 100$ $\mathrm{pm} / \mathrm{V})$ and collagen $(\sim 3 \mathrm{pm} / \mathrm{V})$ are very close to the corresponding macroscopic values.

Finally, AFAM and PFM images of the (100) surface of single crystal $\mathrm{BaTiO}_{3}$ are illustrated in Fig. 7. In this case, the effect of surface topography, contaminants, and crystallographic variations on PFM and AFAM images is minimized, allowing more quantitative studies. The topographic image reveals characteristic corrugations due to the presence of bulk $90^{\circ}$ domain walls. Because of selective etching during sample preparation, the surface morphology exhibits a number of smaller topographic features (watermarks) which result in formation of surface-specific domains clearly visible on the PFM image. The combination of PFM amplitude and phase images allows the local domain structure to be identified. ${ }^{19}$ The PFM image contains large non-local contributions due to electrostatic forces inevitable for the soft $(k$ $=0.5 \mathrm{~N} / \mathrm{m}$ ) cantilevers used in this case. The effective PFM signal defined as half the amplitude difference between $c^{+}$ and $c^{-}$domains, is $\sim 30 \mathrm{pm} / \mathrm{V}$, as compared to the calculated value of $C_{3}^{*} / C_{1}^{*}=36 \mathrm{pm} / \mathrm{V}$ for $\mathrm{BaTiO}_{3}$. AFAM images 


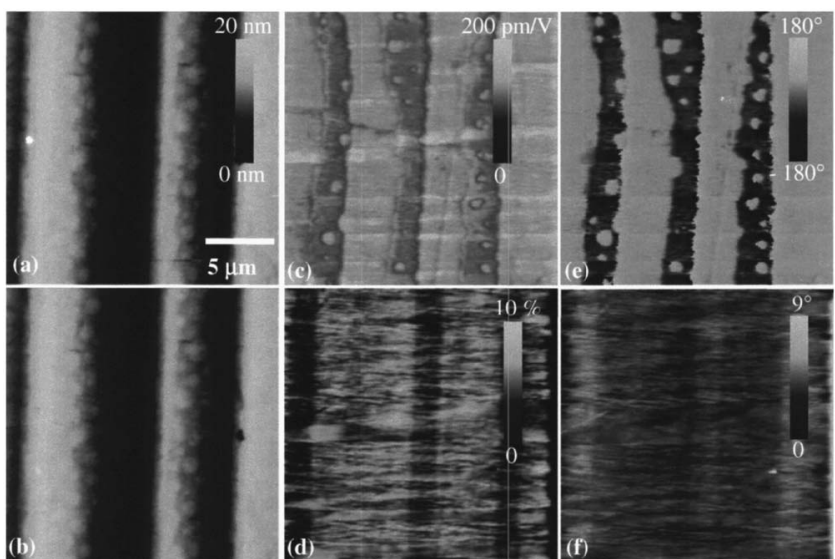

FIG. 7. Surface topography (a,b) PFM amplitude (c), AFAM amplitude (d), PFM phase (e), and AFAM phase (f) images on the $\mathrm{BaTiO}_{3}$ (100) surface with $a-c$ domain structure. Note that in the AFAM image only $a$ and $c$ domains can be distinguished and the noise level due to topographic crosstalk is extremely high, whereas in the PFM image $c^{+}$and $c^{-}$domains are distinguished as well. However, as described in the text, lateral resolution is ultimately expected to be higher for AFAM.

show a clear contrast between $a$ - and $c$-domains of the order of $\sim 2 \%$ of the average signal. At the same time, there is no visible contrast between regions with $c^{+}$and $c^{-}$domain orientation. The AFAM contrast observed can be attributed either to a difference in adhesion forces between the tip and the surface due to variation in surface composition, ${ }^{20}$ or to a difference in local elastic moduli. It was shown by Kalinin et $a l .{ }^{21,22}$ that the surface chemistry of $\mathrm{BaTiO}_{3}$ (100) is strongly dependent on polarization orientation, suggesting that if the difference of the adhesion force was the dominant contribution in AFAM images, regions with different normal components of polarization would be distinguishable. Rabe et $\mathrm{al}^{11}$ has estimated that the local polarization orientation gives rise to a significant $\left(M_{i p}=213 \mathrm{GPa}, M_{\text {oop }}=159.8 \mathrm{GPa}\right)$ difference in local elastic properties between in-plane and out-of-plane domains. Observed contrast between $a$ and $c$ domains (in-plane domains are brighter in the AFAM amplitude image, consistent with a higher indentation modulus) and lack of contrast between $c^{+}$and $c^{-}$domains suggest that the local elasticity dominates the AFAM signal.

These examples illustrate that PFM and AFAM provide complimentary information on local elastic and electromechanical properties that provides a comprehensive understanding of local material functionality. Here, we analyze the detection mechanism in PFM and AFAM in the lowfrequency limit and analyze their resolution and sensitivity to detailed geometry of tip-surface junction.

Quantitative interpretation of elastic and electromechanical data requires a unified theoretical description of piezoacoustic SPM including cantilever dynamics and tip-surface contact mechanics. Schematics illustrating the image formation mechanism for PFM and AFAM are shown in Fig. 8. In the low-frequency regime when the inertial effects are minimal and dynamic cantilever stiffening can be ignored, the complete description of PFM and AFAM imaging mechanism for transversally isotropic piezoelectric material can be

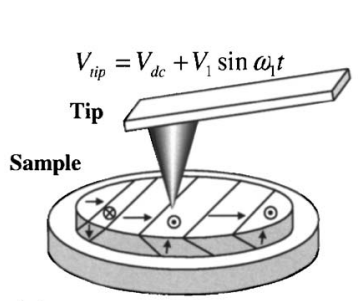

(a)
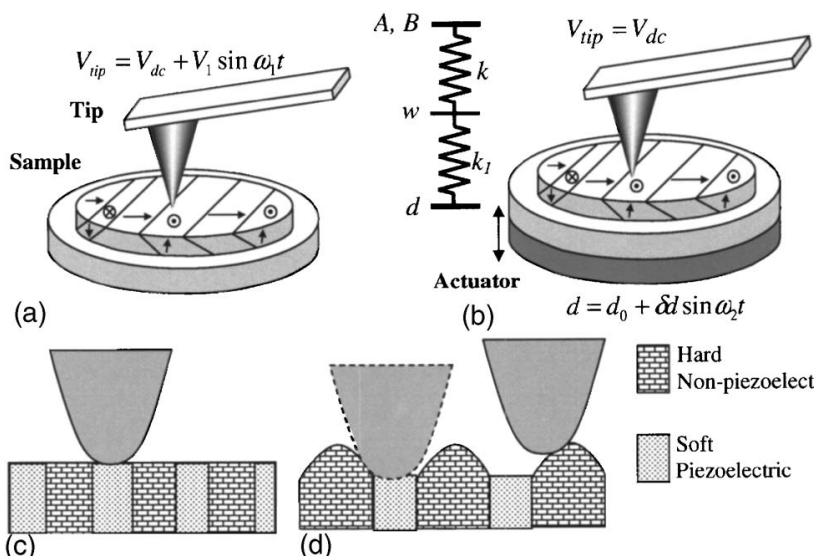

臣 Hard Non-piezoelectric Soft Piezoelectric

FIG. 8. Schematics of the contrast formation mechanism for PFM (a) and AFAM (b). Also shown is the equivalent mechanical circuit for the tipsurface junction and cantilever. Signal in AFAM is determined by the spring constant of the tip-surface junction, directly proportional to contact radius. Thus, regions with different mechanical properties can be unambiguously distinguished only in the absence of topographic variations (c), while on topographically inhomogeneous surface (d) changes in contact area due to local curvature will affect AFAM signal. In comparison, PFM signal is independent on contact area and thus is insensitive to topographic cross-talk.

obtained using the stiffness relations for a spherical indenter, ${ }^{9}$

$$
P=\frac{2 R^{1 / 2}}{\pi}\left(\frac{2}{3} w_{0}^{3 / 2} C_{1}^{*}+w_{0}^{1 / 2} \psi_{0} C_{3}^{*}\right),
$$

where $w_{0}$ is the indentation depth, $R$ is the indenter radius, $\psi_{0}$ is the indenter potential, and $P$ is the load. The relationship between the contact radius, $a$, and the indentation depth is $a=\sqrt{w_{0} R}$.

The electromechanical PFM signal $A$ includes the amplitude $\delta A$ and phase $\varphi_{1}$ of the tip vibration, $A=A_{0}$ $+\delta A \sin \left(\omega_{1} t+\varphi_{1}\right)$, induced by the periodic bias $V_{\mathrm{tip}}=V_{\mathrm{dc}}$ $+V_{1} \sin \omega_{1} t$ applied to the tip. In contact mode operation, the mechanical load on the tip-surface junction is equal to the elastic force exerted by the tip, $P=k A_{0}$, where $k$ is the spring constant of the cantilever and $A_{0}$ is static set-point cantilever deflection. Since the modulation frequency, $\omega_{1}$, is typically selected much higher than the bandwidth of the feedback loop, the static component of tip deflection is constant. Application of a periodic bias to the tip results in a surface displacement, $w=w_{0}+\delta w \sin \left(\omega_{1} t+\varphi_{1}\right)$, where the static indentation depth, $w_{0}$, for a given load, $P$, and dc component of the tip potential, $V_{\mathrm{dc}}$, can be found from Eq. (1) for $\psi_{0}$ $=V_{\mathrm{dc}}$. For small bias amplitudes, Eq. (1) can be linearized as $\delta P=k_{1} \delta w$, where $k_{1}$ is the bias-dependent spring constant of the tip-surface junction, $k_{1}=R^{1 / 2}\left(2 w_{0}^{1 / 2} C_{1}^{*}-w_{0}^{-1 / 2} \psi_{0} C_{3}^{*}\right) / \pi$. From force balance, $\delta A k=-(\delta A-\delta w) k_{1}$ and the tip displacement is related to a change in the indentation depth as

$$
\delta A=\frac{k_{1}}{k+k_{1}} \delta w .
$$

For a typical ferroelectric, $\mathrm{BaTiO}_{3}$, in the $c^{+}$domain state, $C_{1}^{*}=403 \mathrm{GPa}, C_{3}^{*}=15.4 \mathrm{~N} / \mathrm{V} \mathrm{m}$ and for a tip with $R$ $=50 \mathrm{~nm}$ and $P=200 \mathrm{nN}$ for $\psi_{0}=0$ the indentation depth is 
found to be $w_{0}=3.01 \AA$ and the effective tip-surface spring constant is $k_{1}=993 \mathrm{~N} / \mathrm{m}$. This is significantly higher than the typical cantilever spring constant $k \sim 1-50 \mathrm{~N} / \mathrm{m}$. Hence, the tip deflection is almost equal to the surface displacement, $\delta A \approx \delta w$, which is the usual assumption in PFM. Surface displacement, in turn, is directly related to the tip potential and for zero dc bias, $\delta w=V_{1} C_{3}^{*} / C_{1}^{* 9}$, relating the displacement directly to the electromechanical properties of the surface. It is well known that PFM contrast is strongly affected by nonlocal electrostatic forces which produce buckling oscillations of the cantilever. ${ }^{23}$ Minimization of this contribution requires imaging with stiff cantilevers with $k$ $>1-10 \mathrm{~N} / \mathrm{m}$. Equation (2) illustrates that excessively stiff cantilevers will reduce the PFM signal, since $k_{1} \sim P^{1 / 3}$ $\sim k^{1 / 3}$ and for $k \rightarrow \infty$ the PFM signal will vanish, $\delta A \rightarrow 0$. However, simple estimates suggest that surface and tip wear will become a problem long before this limit is achieved.

The elastic AFAM signal, $B$, includes the amplitude, $\delta B$, and phase, $\varphi_{2}$, of the cantilever oscillation, $B=B_{0}$ $+\delta B \sin \left(\omega_{2} t+\varphi_{2}\right)$, where $B_{0}$ is the static cantilever deflection. Cantilever oscillations are induced by the periodic mechanical oscillations of the sample, $d=d_{0}+\delta d \sin \omega_{2} t$, generated by the piezoelectric transducer. In this case, the displacement of the sample base, $\delta d$, results in a mechanical tip displacement, $\delta B$. The two displacements are generally different and the corresponding change in the indentation force, $\delta P$, is balanced by a change in the mechanical force exerted by the cantilever $\delta F=k \delta B$, where $k$ is the cantilever spring constant. From simple mechanical considerations, the change in the indentation depth is $\delta w=\delta d-\delta B$ and the relationship between the sample displacement and the change in indentation depth is $\delta w=\delta d k /\left(k+k_{1}\right)$. The corresponding change in the indentation force is $\delta P=\delta d k k_{1} /\left(k+k_{1}\right)$. The tip displacement is $\delta B=\delta d k_{1} /\left(k+k_{1}\right)$. For $k_{1} \gg k$, the AFAM signal can be approximated as $\delta B=\delta d\left(1-k / k_{1}\right)$. Given that $k_{1}$ $\sim k^{1 / 3}$, AFAM imaging is possible only for small indentation forces corresponding to small tip surface spring constants, $\mathrm{k}_{1} \cdot{ }^{24}$ Note that the tip-surface spring constant for zero dc bias, $k_{1}=0.847 R^{1 / 3}\left(C_{1}^{*}\right)^{2 / 3} P^{1 / 3}$, is weakly dependent on tip properties and indentation force and the expected AFAM contrast for a $\mathrm{BaTiO}_{3}$ surface is $\sim 0.03 \%-0.1 \%$, i.e., an order of magnitude lower than the observed value. We ascribe this discrepancy to the large modulation amplitude which limits applicability of the linear approximation and also accounts for the strong contrast on AFAM phase images in Figs. 2-4. An alternative explanation for this behavior is tip flattening, where the tip-surface contact spring constant, $k_{1}$ $=2 a C_{1}^{*} / \pi$, where $a$ is contact radius, is force-independent. For small contact radii, of order of $1-3 \mathrm{~nm}, k_{1}$ $\sim 250-750 \mathrm{~N} / \mathrm{m}$, resulting in higher AFAM contrast. Notably, maximization of the AFAM signal requires imaging at small indentation forces using soft cantilevers. However, in ambient conditions the capillary forces impose a lower limit on the tip-surface force, necessitating the use of highfrequency UFM or AFAM modes.

The key difference between PFM and AFAM is the different sensitivity to the topographic cross-talk, namely the variations in radius of contact due to local surface curvature [Figs. 8(c) and 8(d)]. In PFM, electromechnical surface displacement $\delta w=V_{1} C_{3}^{*} / C_{1}^{*}$ is independent on contact radius, whereas in AFAM the spring constant of the tip surface junction, $k_{1}=2 a C_{1}^{*} / \pi$, is linear in contact radius. This implies that topographic features such as grooves, ridges, etc. will affect PFM signal only weakly, whereas they will strongly affect AFAM contrast. As illustrated for the materials above, experimentally measured PFM amplitudes are well within the limits expected to the particular class of materials. This is particularly important for biological or inorganic systems that typically exhibit complex surface topography, e.g., compare Figs. 4(c) and 4(d). While the acoustic image is strongly related to topographic structure, PFM images are relatively topography independent, unless the contact with the tip side occurs. The influence of surface slope on AFAM and PFM data will be analyzed elsewhere.

One of the outstanding questions in AFAM and PFM imaging is the signal generation volume, which determines both lateral resolution and depth sensitivity. Both techniques are ultimately sensitive to bias and displacement-induced changes in the indentation depth. Thus, the signal generation volume in PFM is given by $\delta u_{z} / \delta V$, whereas in AFAM the signal generation volume is $\delta u_{z} / \delta d$, where $u_{z}=u_{z}(\rho, z)$ is the normal displacement field below the tip and $\delta u_{z}$ is a change in displacement due to variation in tip potential, $\delta V$, or sample position, $\delta d$. In Refs. 9 and 10, the complete structure of the electroelastic field below the SPM tip was analyzed. It was shown that the normal displacement field could be represented as a linear superposition of the fields $u_{z}=u_{z, m}(a)$ $+u_{z, e}^{0}(a) \psi_{0}$. Here $u_{z, m}$ is the solution of the indentation problem with purely mechanical boundary conditions and zero potential and $u_{z, e}^{0}(a, R) \psi_{0}$ is the solution of the purely electrical problem. Electrical and mechanical modulations applied to the tip or to the sample change the indentor bias and contact radius, thus affecting corresponding fields. In PFM, the indentation force is constant, $P=$ const, and from Eq. (1) the change in the tip bias, $V=\psi_{0}+\delta V$, results in a change of contact area, $a=a_{0}+\delta a_{\mathrm{PFM}}$, such that

$$
\delta a_{\mathrm{PFM}}=\delta V\left(\frac{2 a_{0}}{R} \frac{C_{1}^{*}}{C_{3}^{*}}+\frac{\psi_{0}}{a_{0}}\right)^{-1} .
$$

In AFAM, the tip bias is constant, $V=$ const, and modulation in the sample position is equivalent to a change in the indentation force, $P=k A+\delta P$, where $\delta P=\delta d k k_{1} /\left(k+k_{1}\right)$. This results in a change of contact area, $a=a_{0}+\delta a_{\mathrm{AFAM}}$, such that

$$
\delta a_{\mathrm{AFAM}}=\delta P\left(\frac{8 a_{0}^{2} C_{1}^{*}}{3 \pi R}+\frac{2 \psi_{0} C_{3}^{*}}{\pi}\right)^{-1} .
$$

The change in the displacement field distribution $\delta u_{z}$ $=u_{z}\left(a+\delta a_{\mathrm{PFM}}, \psi_{0}+\delta V\right)-u_{z}\left(a, \psi_{0}\right)$ thus determines a signal generation volume in PFM and $\delta u_{z}=u_{z}\left(a+\delta a, \psi_{0}\right)$ $-u_{z}\left(a, \psi_{0}\right)$ determines a signal generation volume in AFAM. The calculated signal generation volumes for PFM and AFAM are shown in Fig. 9. The spatial extent of the displacement fields inside the material for both PFM and AFAM 


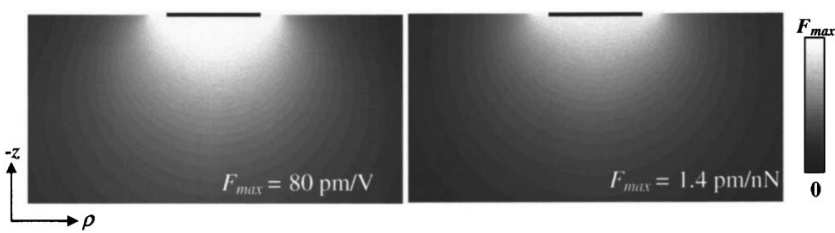

FIG. 9. Signal generation volume for PFM (a) and AFAM (b) calculated for PZT6b. The brightness indicates the magnitude of the corresponding field. Note that for both elastic and electromechanical imaging, the signal generation volume and hence resolution is ultimately determined by the tip-surface contact area. However, while vibration transfer through air is negligible for AFAM, in PFM the electrostatic field produced by the spherical and conical parts of the tip not in contact with the surface result in a decrease of effective resolution.

is determined by the contact radius between the tip and the surface, which thus imposes the limit on achievable resolution. The strain field in AFAM is determined only by the tip-surface contact and vibration transfer through air should be negligible. In contrast, in PFM an additional contribution to the electrostatic field inside the material and hence the displacement field is produced by the part of the tip not in contact with the surface. A measure of the nonlocal contribution to the signal can be obtained from the effective image resolution.

This simple consideration suggests that AFAM resolution on ferroelectric materials will always be higher than PFM. However, AFAM allows only domains with nonparallel polarization components to be distinguished, whereas PFM distinguishes collinear (antiparallel) domains as well. In addition, AFAM is extremely sensitive to surface topography, while PFM is relatively topography insensitive, thus imposing further limits on applicability of AFAM for quantitative ferroelectric imaging. Both PFM and AFAM contrast will be strongly affected by the presence of surfaces contaminates, which can be particularly important for biological systems.

To summarize, we have demonstrated an approach for simultaneous elastic and electromechanical imaging by PASPM and illustrated its applicability to ferroelectric materials and biological systems. Given the restrictions imposed on the measurements of linear tip-surface capacitance, simultaneous measurements of local indentation stiffness and indentation piezocoefficient provide a comprehensive description of the local electroelastic properties for transversally isotropic material. The contrast formation mechanism in AFAM and PFM in the low-frequency regime is described in terms of tipsurface contact mechanics. The cantilever response and signal generation volumes for both techniques are determined. PFM imaging requires stiff cantilevers, whereas AFAM signal can be maximized for soft cantilevers to reduce the spring constant of the tip-surface junction. Alternatively, high-frequency modes such as UFM can be used in direct conjunction with PFM measurements.

Research was performed as a Eugene P. Wigner Fellow and staff member at the Oak Ridge National Laboratory, managed by UT-Battelle, LLC, for the U.S. Department of Energy under Contract No. DE-AC05-00OR22725 (S.V.K.). Support of ORNL SEED funding (A.P.B., E.K., and S.V.K.) is acknowledged. A.G. acknowledges financial support of the National Science Foundation (Grant No. DMR02-35632) and Bilateral US-Israel Science Foundation. Sam Snyder (PiezoKinetics) is gratefully acknowledged for the piezoactuator samples. Research is partially supported by NSF (M.K. and E.K. Grant No. CMS-0509936).

${ }^{1}$ U. Rabe, V. Sherer, S. Hirsekorn, and W. Arnold, J. Vac. Sci. Technol. B 15, 1506 (1997).

${ }^{2}$ N. A. Burnham, A. J. Kulik, G. Gremaud, P.-J. Gallo, and F. Oulevey, J. Vac. Sci. Technol. B 14, 794 (1996).

${ }^{3}$ P. Maivald, H. J. Butt, S. A. C. Gould, C. B. Prater, B. Drake, J. A. Gurley, V. B. Elings, and P. K. Hansma, Nanotechnology 2, 103 (1991).

${ }^{4}$ S. A. Syed Asif, K. J. Wahl, R. J. Colton, and O. L. Warren, J. Appl. Phys. 90, 1192 (2001).

${ }^{5}$ K. Yamanaka, H. Ogiso, and O. Kolosov, Appl. Phys. Lett. 64, 178 (1994).

${ }^{6}$ Applied Scanning Probe Methods, edited by B. Bhushan, H. Fuchs, and S. Hosaka (Springer, Berlin, 2004).

${ }^{7}$ Nanoscale Characterization of Ferroelectric Materials, edited by M. Alexe and A. Gruverman (Springer, Berlin, 2004).

${ }^{8}$ C. Halperin, S. Mutchnik, A. Agronin, M. Molotskii, P. Urenski, M. Salai, and G. Rosenman, Nano Lett. 4, 1253 (2004).

${ }^{9}$ S. V. Kalinin, E. Karapetian, and M. Kachanov, Phys. Rev. B 70, 184101 (2004).

${ }^{10}$ E. Karapetian, M. Kachanov, and S. V. Kalinin, Philos. Mag. 85, 1017 (2005).

${ }^{11}$ U. Rabe, M. Kopycinska, S. Hiserkorn, J. Munoz-Saldana, G. A. Schneider, and W. Arnold, J. Phys. D 35, 2621 (2002).

${ }^{12}$ SD 0.394-0.000-0.040-502, Piezo Kinetics, Bellefonte, PA 16823 , www.piezo-kinetics.com.

${ }^{13}$ U. Rabe, "Atomic Force Acoustic Microscopy," in Applied Scanning Probe Methods, edited by B. Bhushan and H. Fuchs (to be published).

${ }^{14} \mathrm{~S}$. Jesse et al. (to be submitted).

${ }^{15}$ E. Fukada, Biorheology 32, 593 (1995).

${ }^{16}$ A. A. Marino and R. O. Becker, Nature (London) 228, 473 (1970).

${ }^{17}$ E. Fukada, J. Phys. Soc. Jpn. 10, 149 (1955).

${ }^{18}$ C. A. L. Bassett, Calcif. Tissue Res. 1, 252 (1968).

${ }^{19}$ S. V. Kalinin and D. A. Bonnell, Phys. Rev. B 63, 125411 (2001).

${ }^{20}$ O. V. Kolosov, M. R. Castell, C. D. Marsh, G. A. D. Briggs, T. I. Kamins, and R. S. Williams, Phys. Rev. Lett. 81, 1046 (1998).

${ }^{21}$ S. V. Kalinin, C. Y. Johnson, and D. A. Bonnell, J. Appl. Phys. 91, 3816 (2002).

${ }^{22}$ S. V. Kalinin, D. A. Bonnell, T. Alvarez, X. Lei, Z. Hu, R. Shao, and J. H. Ferris, Adv. Mater. (Weinheim, Ger.) 16, 795 (2004).

${ }^{23}$ S. V. Kalinin and D. A. Bonnell, in Nanoscale Characterization of Ferroelectric Materials, edited by M. Alexe and A. Gruverman (SpringerVerlag, Berlin, 2004).

${ }^{24}$ Alternatively, the use of the inertia effect in high-frequency AFAM or UFM allows the effective stiffness of the cantilever to be increased. 